\title{
Parâmetros genéticos da taxa de maturação e do peso assintótico de fêmeas da raça Nelore
}

[Genetic parameters of maturation rate and asymptotic weight of Nelore females]

\author{
A. Del V. Garnero ${ }^{1}$, C.R. Marcondes $^{2}$, L.A.F. Bezerra ${ }^{2}$, H.N. Oliveira ${ }^{3}$, R.B. Lôbo ${ }^{2}$ \\ ${ }^{1}$ Ciências Biológicas - Universidade Federal de Tocantins \\ Caixa Postal 25 \\ 77500-000 - Porto Nacional, TO \\ ${ }^{2}$ Faculdade de Medicina de Ribeirão Preto - USP - Ribeirão Preto, SP \\ ${ }^{3}$ Universidade Estadual Paulista - Botucatu, SP
}

\begin{abstract}
RESUMO
Utilizaram-se 14.563 pesagens de 1158 fêmeas da raça Nelore, nascidas entre 1984 e 1995, pertencentes a 10 fazendas, distribuídas em sete estados do Brasil. Com o objetivo de estabelecer um padrão médio de crescimento, obter parâmetros individuais das curvas e estimar os componentes de variância, herdabilidade e correlações genéticas dos parâmetros das curvas, foram comparados os modelos de Von Bertalanffy, Brody, logístico e Gompertz. Foram utilizados o procedimento NLIN e o programa MTDFREML sob modelo animal em análise unicaráter e bicaráter. Os parâmetros médios dos pesos assintóticos (A) e das taxas de maturidade (K) foram: 515,06 e 0,071 para Von Bertalanffy; 552,77 e 0,045 para Brody; 501,11 e 0,097 para logístico, e 507,00 e 0,083 para Gompertz, respectivamente. As estimativas de herdabilidade para A e K foram de alta magnitude: 0,39 e 0,42 para Von Bertalanffy, 0,42 e 0,44 para Brody, 0,40 e 0,41 para logístico e 0,39 e 0,39 para Gompertz, respectivamente. As correlações genéticas variaram entre $-0,69$ e $-0,49$. Todos os modelos foram adequados para descrever o crescimento. A ordem de escolha do melhor modelo para descrever a curva de crescimento foi: Brody, Von Bertalanffy, logístico e Gompertz. Essas características seriam passíveis de inclusão em índice de seleção para seleção de fêmeas Nelore.
\end{abstract}

Palavras-chave: bovino, Nelore, curva de crescimento, peso assintótico, taxa de maturação

\begin{abstract}
Data from 1158 females Nellore beef cattle, born between 1984 and 1995, at 10 breeding farms and located at seven differents States of Brazil were used to establish a growth pattern curve of beef cattle females, and to estimate variance components, heritabilities and genetic correlations between the parameters for Von Bertalanffy, Brody, logistic and Gompertz models. The NLIN procedure and the MTDFREML program under animal model were used for single and two trait analyses. The average weight (A) and growth rate $(K)$ were 515.06 and .071 for Von Bertalanffy; 552.77 and .045 for Brody; 501.11 and .097 for logistic; and 507.00 and .083 for Gompertz, respectively. High heritabilities were estimated for $A$ and K parameters: .39 and .42 for Von Bertalanffy; .42 and .44 for Brody; .40 and .41 for logistic; and .39 and .39 for Gompertz, respectively. All the models described adequately the growth pattern of those females. The best model to describe the growth curve based on the number of iteractions for convergence, error mean square and the coefficient of determination was Brody model, followed by Von Bertalanffy, logistic and Gompetz models. These characteristics (A and $K$ parameters) could be included in selection index for female Nelore selection.
\end{abstract}

Keywords: bovine, asymptotic weight, growth rate, growth curves

Recebido para publicação, após modificações, em 29 de novembro de 2004

E-mail: analiagarnero@uft.edu.br 


\section{INTRODUÇÃO}

O aumento do peso vivo ao longo da vida do animal é fenômeno complexo e dependente do genótipo e dos efeitos ambientais que atuam ao longo do tempo.

Em bovinos, as maiorias das pesquisas são voltadas para as etapas iniciais do crescimento, limitando-se às medidas em idades-padrão como à desmama, ao ano e ao sobreano. Esse tipo de seleção geralmente resulta em aumento do peso dos animais adultos, que eleva os custos de manutenção das vacas no rebanho (Cartwright, 1970; Lanna e Packer, 1997) e, conseqüentemente, reduz as vantagens econômicas do aumento ao peso dos animais de abate. Rosa et al. (2001) observaram, na raça Nelore, grande variabilidade genética do tamanho adulto e herdabilidade de magnitude mediana $(0,34)$, concluindo não ser assunto de preocupação, se considerado como critério de seleção. Cartwright (1970) apontou a seleção para alterar a forma da curva de crescimento como uma das maneiras de se conseguir animais que atinjam o peso de abate mais cedo sem aumentar o tamanho dos animais.

Zebuínos em geral apresentam, em relação aos taurinos, menor velocidade de crescimento. Contudo, são poucos os trabalhos com pesos de todo o ciclo de vida dos zebuínos, desde o nascimento até a maturidade, como o de Oliveira et al. (2000), que estudaram o crescimento de fêmeas Guzerá com dados após 60 meses de idade. A maioria dos estudos utilizou dados de animais cujas pesagens foram interrompidas antes que a maturidade fosse atingida (Cortarelli et al., 1983; Santoro e Barbosa, 2001), portanto, não existe uma descrição global do crescimento e nem foi estabelecido o que pode ser considerado como padrão médio de crescimento para zebuínos, mais precisamente, da raça Nelore.

Em estudos sobre curva de crescimento, as pesagens são usadas para descrever a curva, e seus parâmetros são estimados com a finalidade de usá-los como critérios de seleção. Segundo Fitzhugh (1976), a possibilidade de alterar geneticamente a curva de crescimento depende do grau de flexibilidade genética da forma da curva, sugerindo a utilização de um índice que inclua características de tamanho e maturidade. Entretanto, Oliveira et al. (2000) concluíram que seria difícil alterar a curva de crescimento na raça Guzerá por meio da seleção praticada sobre os parâmetros da curva, uma vez que a taxa de maturação e o peso assintótico apresentam correlações (genética, ambiental e fenotípica) altas e negativas.

Existe variabilidade de recursos genéticos, tanto em zebuínos como em taurinos, que poderia atender às diferentes demandas dos sistemas de produção, das condições ambientais e dos recursos disponíveis, evidenciando que há um tamanho ótimo para cada necessidade (Stewart e Martin, 1983; Rosa et al., 2001; Arango e Van Vleck, 2002). Contudo, é necessário considerar características de crescimento e maturidade derivadas do estudo de curvas de crescimento como informação adicional em programas de melhoramento genético (Kaps et al., 2000; Arango e Van Vleck, 2002).

Modelos matemáticos não lineares, desenvolvidos empiricamente para relacionar peso e idade, têm se mostrado adequados para descrever a curva de crescimento. Em bovinos, os cinco mais utilizados são: Brody, Von Bertalanffy, Richards, logístico e Gompertz (Oliveira, 1995). Nas raças taurinas, o uso dos modelos já é bem conhecido para descrever o processo de crescimento das fêmeas e, geralmente, os modelos Richards e Brody são os que se mostram mais adequados. Em zebuínos, mais particularmente na raça Guzerá, Oliveira et al. (2000) concluíram que os modelos Von Bertalanffy, Brody, logístico e Gompertz foram adequados para descrever o crescimento em fêmeas, sendo o primeiro o mais indicado.

Os objetivos deste trabalho foram estabelecer um padrão médio de crescimento, obter parâmetros individuais e médios das curvas, estimar os componentes de (co)variância, as herdabilidades e as correlações genéticas dos parâmetros A e K e comparar os modelos Brody, Von Bertalanffy, logístico e Gompertz, utilizando dados de crescimento até a maturidade de fêmeas da raça Nelore.

\section{MATERIAL E MÉTODOS}

Foram utilizados 14.563 registros de pesos e idades referentes a 1158 fêmeas da raça Nelore (Fig. 1), nascidas entre 1984 e 1995, 
provenientes de 10 fazendas participantes do Programa de Melhoramento Genético da Raça Nelore (PMGRN), e distribuídas pelos estados da
Bahia ( $n=11)$, Goiás ( $n=73)$, Mato Grosso do Sul $(\mathrm{n}=476)$, Minas Gerais $(\mathrm{n}=320)$, Pará $(\mathrm{n}=3)$, Paraná (n=2) e São Paulo ( $n=273)$.

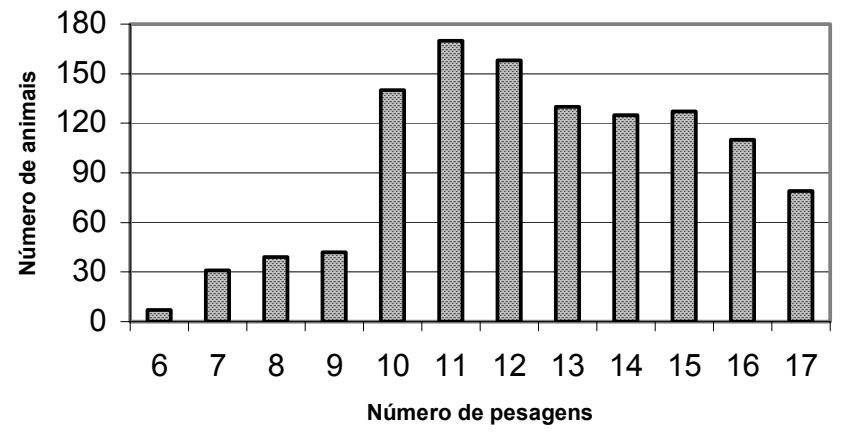

Figura 1. Número de pesagens por animal da raça Nelore, durante o ciclo de vida, no Programa de Melhoramento Genético da Raça Nelore.

Os animais participantes do PMGRN, além de serem identificados de forma a garantir as informações de genealogia, foram submetidos a mensurações trimestrais. Os produtos foram monitorados do nascimento aos 21 meses de idade, e as matrizes pesadas ao parto, à desmama dos seus produtos e nos meses de abril e outubro de cada ano. As fazendas adotaram estação de monta, cuja duração variou entre 60 e 90 dias, entre outubro e março, conforme a região e o ano. A utilização da inseminação artificial foi freqüente. A desmama dos bezerros foi feita, geralmente, aos sete - oito meses de idade.

Para descrever o padrão médio de crescimento e os parâmetros individuais das fêmeas da raça Nelore, foram utilizados quatro modelos matemáticos não lineares (Tab. 1). Nas expressões matemáticas, Y representa o peso do animal a uma determinada idade $(\mathrm{t})$, A é o valor assintótico de Yt (peso médio à maturidade), B é a constante de integração relacionada com os pesos iniciais (grau de maturidade do animal ao nascimento), $\mathrm{K}$ é a taxa de variação da função exponencial (velocidade com a qual o animal se aproxima ao tamanho adulto), $\mathrm{M}$ é o parâmetro que dá forma à curva, $e$ é o logaritmo em base natural e $\varepsilon$ representa o erro aleatório associado a cada pesagem.

Foram calculadas as idades aos 50 e $95 \%$ de maturação e os pontos de inflexão para cada modelo (Tab. 2).

Tabela 1. Modelos não lineares utilizados para descrever o padrão médio de crescimento e parâmetros individuais das fêmeas da raça Nelore

\begin{tabular}{lcc}
\hline Modelo & Autor $^{1}$ & Equação \\
\hline Brody & (Brody, 1945) $\left.^{-K t}\right)+\varepsilon$ \\
Von Bertalanffy & (Bertalanffy, 1957) & $Y_{t}=A\left(1-B e^{-K t}+Y_{t}=A\left(1-B e^{-K t}\right)^{3}+\varepsilon\right.$ \\
Logístico & (Nelder, 1961) & $Y_{t}=A\left(1+e^{-K t}\right)^{-M}+\varepsilon$ \\
Gompertz & (Laird, 1966) & $Y_{t}=A e^{-B e^{\wedge}(-K t)}+\varepsilon$ \\
\hline
\end{tabular}

Oliveira (1995). 
Tabela 2. Equações para cálculo da idade aos 50\% de maturação e pontos de inflexão para cada modelo

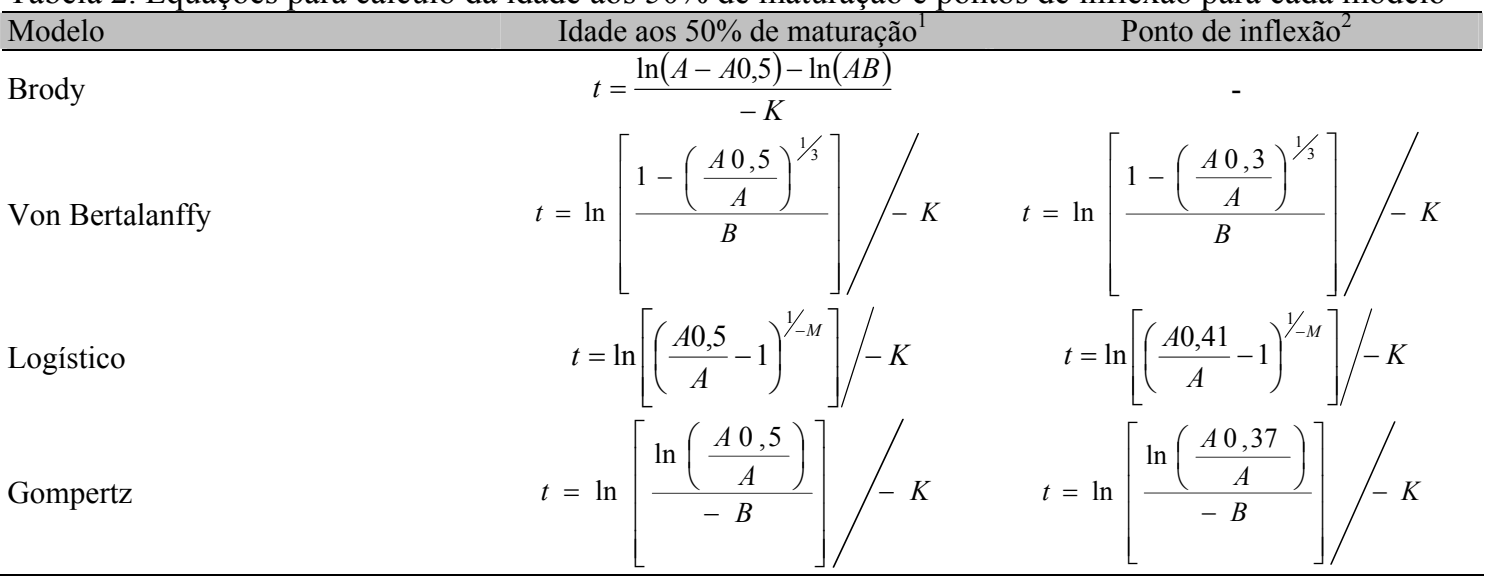

${ }^{1}$ Para obter a idade aos $95 \%$ de maturação, mudar nas equações 0,5 por 0,95 .

${ }^{2} \mathrm{O}$ ponto de inflexão para o modelo Brody é o próprio peso ao nascimento.

Foi utilizado o procedimento NLIN do SAS (Statistical... 1996) para a obtenção das estimativas individuais dos parâmetros e dos parâmetros médios a partir da totalidade dos dados disponíveis.

A comparação dos modelos quanto ao ajustamento aos dados foi realizada com base em indicadores simples, dada a natureza das variáveis utilizadas. Assim, foram observados o número de iterações e o quadrado médio do erro, sendo o coeficiente de determinação calculado segundo Sampaio (1998).

Para a obtenção dos componentes de (co)variância e valores genéticos dos pesos assintóticos (A) e das taxas de maturidade (K), utilizou-se o programa Multiple Trait Derivative Free Restricted Maximum Likelikood (MTDFREML; Boldman, 1995), sob modelo animal, em análises unicaráter e bicaráter. $\mathrm{O}$ modelo incluiu como fixos os efeitos de grupo de contemporâneos (GC) e como aleatório, o efeito genético direto.

Os GC foram constituídos por animais nascidos no mesmo estado, no mesmo trimestre do ano (estação $1=$ meses 1 a $3,2=\operatorname{meses} 4$ a $6,3=$ meses 7 a 9, 4= meses 10 a 12), mesmo ano e sob o mesmo regime alimentar ( $1=$ pasto, $2=$ semiconfinado, $3=$ confinado).

Foram eliminados animais que não apresentavam, pelo menos, uma pesagem entre 24 e 36 meses, uma entre 36 e 48 meses e uma após 54 meses de idade. Também foram eliminados todos os GC com uma observação, permanecendo 153 grupos e 1087 registros para as análises. A matriz de parentesco continha 1900 animais.

Em termos matriciais, o modelo animal pode ser descrito como:

$y=X \beta+Z a+\varepsilon$, em que:

$\mathrm{y}=$ vetor das observações de cada característica (parâmetro A e K); X = matriz de incidência dos efeitos fixos (GC); $\beta=$ vetor dos efeitos fixos; $Z$ $=$ matriz de incidência do efeito genético direto de cada animal; $a=$ vetor de efeitos genéticos diretos aleatórios; $\varepsilon=$ vetor de efeitos residuais aleatórios.

As pressuposições acerca da distribuição de $y, a$, e $\varepsilon$ são descritas como:

$$
\left[\begin{array}{l}
y \\
a \\
\varepsilon
\end{array}\right] \approx N M V\left\{\left[\begin{array}{l}
X \beta \\
0 \\
0
\end{array}\right]\left[\begin{array}{ccc}
V & Z G & R \\
G Z^{\prime} & G & 0 \\
R & 0 & R
\end{array}\right]\right\}
$$

em que: $V=Z G Z{ }^{\prime}+R, G=A \sigma^{2}{ }_{a}$ e $R=I \sigma^{2}$ 


\section{RESULTADOS E DISCUSSÃO}

A partir das médias de todos os pesos observados, portanto, de diferentes anos e épocas de nascimento, obteve-se a curva média de crescimento em função da idade (Fig. 2). A forma da curva indica que há um ponto de inflexão em torno dos 10 meses de idade. Entretanto, a puberdade na raça Nelore ocorre, aproximadamente, aos 24 meses, apesar de existirem referências de animais precoces já aos 14 meses (Eler et al., 2002). A média da idade ao primeiro parto no conjunto de dados foi de 36 meses, e seu efeito sobre a curva aparece levemente definido, com elevação seguida por pequena queda na taxa de crescimento. A partir dessa idade, observou-se crescimento mais lento, com ondulações ao longo da linha, representando, cada uma delas, o número de partos da vaca.

Os valores médios dos parâmetros da curva de crescimento estimados para os quatro modelos utilizados (Tab. 3) foram menores que os relatados por Duarte (1975) e maiores que os estimados por Ludwig (1977), Carrijo (1988) e Santoro e Barbosa (2001). Esses estudos foram realizados com séries de pesos por idades interrompidas antes de os animais atingirem a maturidade, portanto as estimativas dos parâmetros poderiam ser consideradas como extrapolações para todos os modelos utilizados.

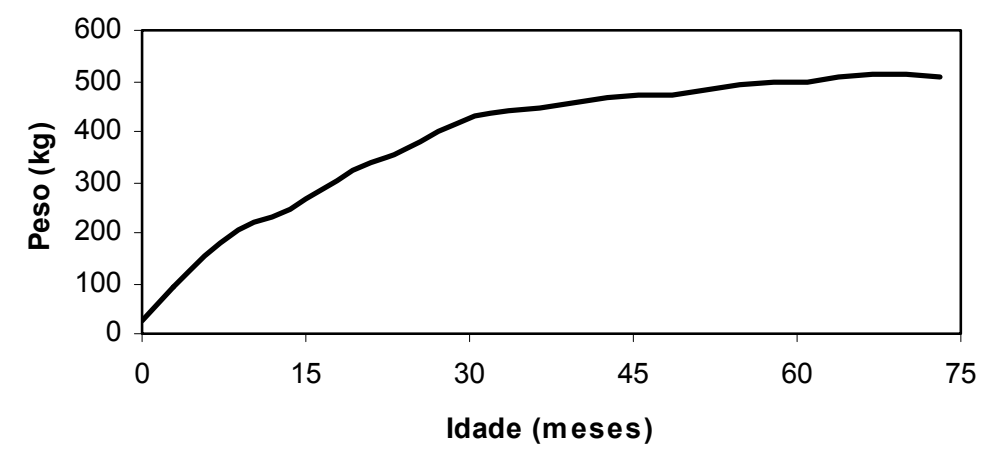

Figura 2. Curva de crescimento obtida a partir das médias dos pesos observados em função da idade de animais do Programa de Melhoramento Genético da Raça Nelore.

Tabela 3. Valores médios, coeficiente de variação (CV), mínimo (Min) e máximo (Max) dos parâmetros da curva de crescimento estimados para os quatro modelos utilizados em fêmeas da raça Nelore

\begin{tabular}{lccccc}
\hline Modelo & Parâmetro & Média & CV & Min & Max \\
\hline \multirow{2}{*}{ Von Bertalanffy } & $\mathrm{A}$ & 515,06 & 12,84 & 353,03 & 844,60 \\
\multirow{3}{*}{ Brody } & $\mathrm{K}$ & 71,00 & 24,76 & 28,00 & 155,90 \\
& $\mathrm{~A}$ & 552,77 & 16,21 & 359,43 & 1054,10 \\
\multirow{2}{*}{ Logístico } & $\mathrm{K}$ & 45,11 & 29,53 & 9,60 & 101,30 \\
& $\mathrm{~A}$ & 501,11 & 12,22 & 348,84 & 811,26 \\
\multirow{2}{*}{ Gompertz } & $\mathrm{K}$ & 97,52 & 22,20 & 44,60 & 205,70 \\
& $\mathrm{~A}$ & 507,27 & 12,44 & 350,94 & 824,77 \\
\hline
\end{tabular}

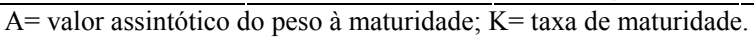

Quando as estimativas dos pesos assintóticos (A) foram comparadas, os valores dos modelos Von Bertalanffy, Gompertz e logístico foram próximos, decrescendo nessa ordem, e inferiores ao estimado pelo modelo Brody. Estes resultados foram semelhantes aos observados em outras raças (Brown et al., 1976; Perotto et al., 1992; Oliveira et al., 2000) e na raça Nelore para o modelo Brody. No entanto, foram superiores às estimativas de Santoro e Barbosa (2001) em 
relação aos modelos Von Bertalanffy, Gompertz e logístico.

Verificou-se grande amplitude nos valores dos parâmetros, indicando a existência de animais com disparidade no peso adulto de aproximadamente $400 \mathrm{~kg}$ e com diferença na velocidade de crescimento, no sentido de atingir o peso assintótico, que chega até 10 meses, dependendo do modelo. Essa constatação confirma a ampla variabilidade em recursos genéticos observada em zebuínos, que poderia atender às diferentes demandas dos sistemas de produção, das condições ambientais e dos recursos disponíveis (Tab. 3).

O parâmetro B representa o grau de maturidade do animal ao nascimento, assim, valores altos representam baixo grau de maturidade ao nascimento. Optou-se por colocar a média do peso ao nascimento das fêmeas da raça Nelore como peso inicial $(28 \mathrm{~kg})$, para não obter um valor de B subestimado. A primeira pesagem, portanto, ocorreu entre o primeiro e o quarto mês de vida. Os valores encontrados de B estão de acordo, como os citados na literatura (Duarte, 1975; Ludwig, 1977; Santoro e Barbosa, 2001), e um pouco menores que os relatados por Oliveira et al. (2000).

O parâmetro $\mathrm{K}$ representa a taxa de maturidade e indica a velocidade de crescimento no sentido de atingir o peso assintótico a partir de seu peso inicial. Quanto maior o valor de $\mathrm{K}$, mais rapidamente o animal se aproxima do seu peso assintótico, ou seja, maior a velocidade de seu crescimento. Animais com alto valor de $\mathrm{K}$ apresentam maturidade precoce em comparação com indivíduos de valor mais baixo de $\mathrm{K}$ e de peso inicial semelhante. Como os pesos iniciais são aproximadamente iguais, a variação de $\mathrm{K}$ representa, com boa precisão, a velocidade relativa de crescimento do animal.

A partir das informações derivadas da curva de crescimento, foram estimadas as médias de idade para se atingir $50 \%$ e $95 \%$ de maturidade e o ponto de inflexão, segundo os modelos utilizados (Tab. 4).

Tabela 4. Médias de idade para atingir 50\% e 95\% de maturidade e ponto de inflexão, segundo os modelos utilizados para fêmeas da raça Nelore

\begin{tabular}{lcccc}
\hline Característica & Von Bertalanffy & Brody & Gompertz & Logístico \\
\hline Idade com 95\% da maturidade & 49,86 & 65,24 & 45,41 & 42,25 \\
Idade com 50\% da maturidade & 13,64 & 14,07 & 13,54 & 13,64 \\
Idade no ponto de inflexão & 6,81 & --- & 9,13 & 10,60 \\
\hline
\end{tabular}

${ }^{1}$ Em meses.

Considerando como adultos os animais que atingiram 95\% do peso assintótico, a idade à maturidade estimada variou consideravelmente conforme o modelo empregado. As idades com $95 \%$ de maturidade estimadas a partir dos modelos Von Bertalanffy, Gompertz e logístico foram mais próximas às encontradas para as raças taurinas (Nadaralah et al., 1984; Beltran et al., 1992; Kaps et al., 2000) e à mencionada por Oliveira et al. (2000) para a raça Guzerá. A idade estimada pelo modelo Brody foi 15 meses superior às estimadas por outros modelos, sendo próxima ao valor verificado por Oliveira et al. (2000), ao utilizarem o mesmo modelo (64 meses). A idade ao se atingir 50\% de maturidade foi semelhante para todos os modelos. Provavelmente essas diferenças refletem problemas nos modelos quanto ao ajustamento no final da curva.

Os pontos de inflexão foram associados a idades com pequena variação entre os modelos, sendo o maior deles estimado a partir do modelo logístico. O ponto estimado pelo modelo Von Bertalanffy correspondeu ao período em que o ganho observado foi máximo (entre dois e seis meses de idade) e pelo modelo Gompertz correspondeu ao segundo intervalo de maior ganho (entre seis e nove meses). Se se relacionar o ponto de inflexão com o início da puberdade, ou seja, a idade em que o animal é capaz de reproduzir, provavelmente todos eles estariam perto da realidade pelo fato de muitas novilhas serem incapazes de reproduzir após a primeira ovulação, ou por não apresentarem sinais 
exteriores de cio e só atingirem a maturidade sexual (fertilidade sexual, fisiológica e comportamental) no terceiro estro (Lanna e Packer, 1997).

Com as curvas de crescimento formadas pelas médias dos pesos observados nas diferentes idades e pelos pesos preditos em relação a essas mesmas idades a partir dos parâmetros estimados, pode-se comparar os ajustamentos de cada modelo aos pesos observados (Fig. 3, 4, 5 e 6). Todos os modelos, em geral, ajustaram-se aos pesos observados. Para os pesos preditos pelo modelo Von Bertalanffy, em quatro fases da curva, houve super ou subestimação (Fig. 3). Pelo modelo Brody (Fig. 4), observou-se subestimação dos valores em torno dos 30 meses de idade, que correspondem aproximadamente à primeira gestação. No entanto, não parece haver superestimação do peso adulto, embora o valor do peso assintótico estimado pelo modelo Brody tenha sido o mais alto. Pelo modelo Gompertz (Fig. 5), dos que melhor se ajustaram aos pesos observados, verificou-se superestimação no começo da curva e subestimação entre seis e 12 meses de idade. No entanto, esse foi o único modelo no qual um animal não atingiu a convergência (Tab. 5). Pelo modelo logístico (Fig. 6), verificou-se super ou subestimação em três fases da curva e menor estimativa do peso adulto (Tab. 3 ).

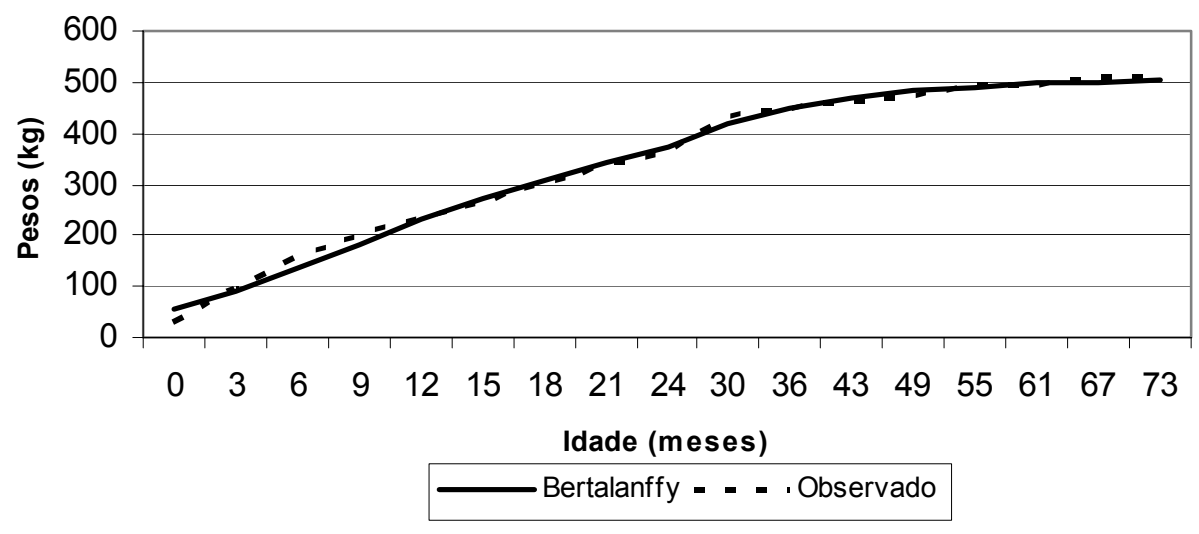

Figura 3. Curvas de crescimento formadas pelas médias dos pesos observados nas diferentes idades e pelos pesos preditos em relação a essas mesmas idades a partir dos parâmetros estimados pelo modelo Von Bertalanffy.



Figura 4. Curvas de crescimento formadas pelas médias dos pesos observados nas diferentes idades e pelos pesos preditos em relação a essas mesmas idades a partir dos parâmetros estimados pelo modelo Brody. 


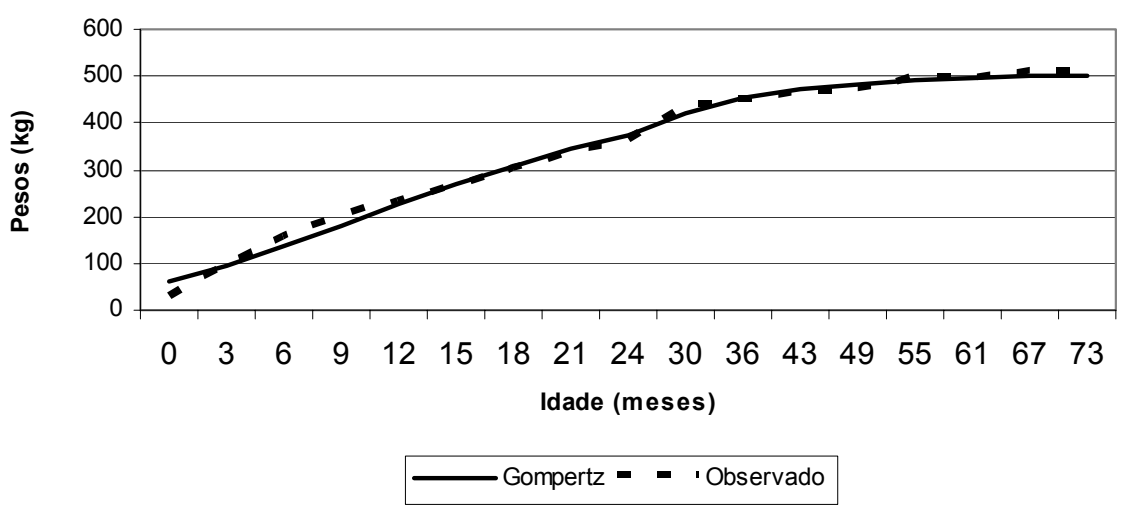

Figura 5. Curvas de crescimento formadas pelas médias dos pesos observados nas diferentes idades e pelos pesos preditos em relação a essas mesmas idades a partir dos parâmetros estimados pelo modelo Gompertz.

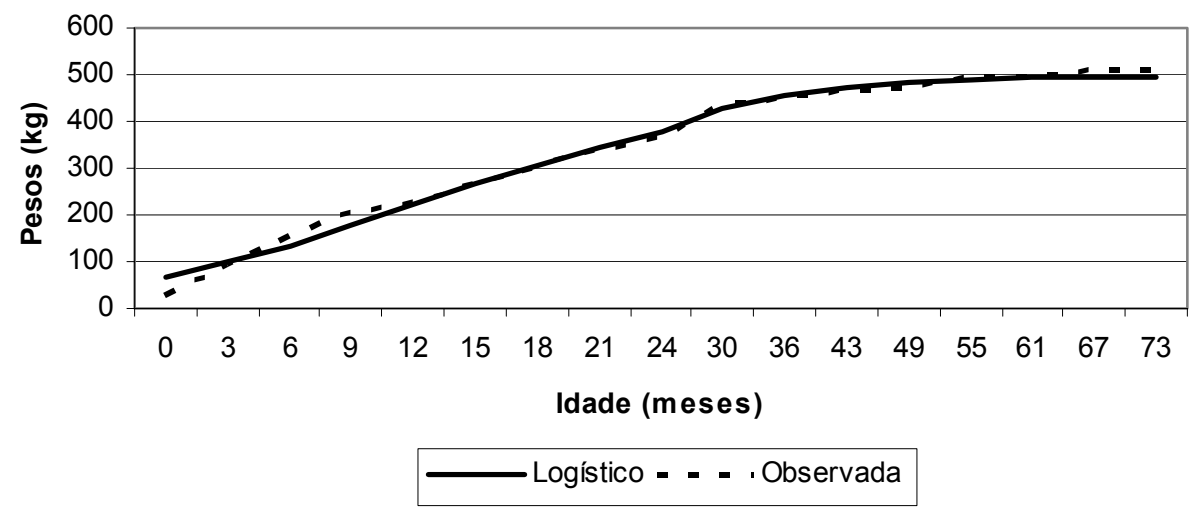

Figura 6. Curvas de crescimento formadas pelas médias dos pesos observados nas diferentes idades e pelos pesos preditos em relação a essas mesmas idades a partir dos parâmetros estimados pelo modelo logístico.

Tabela 5. Critérios de comparação de curvas de crescimento, segundo o modelo adotado para fêmeas da raça Nelore

\begin{tabular}{lcccc}
\hline Critério & Von Bertalanffy & Brody & Gompertz & Logístico \\
\hline $\mathrm{N}^{\circ}$ de iterações & 14 & 10 & $17 \mathrm{a}$ & 13 \\
Quadrado médio do erro & 2633,8 & 2572,7 & 2696,1 & 2763,3 \\
Coeficiente de determinação & 0,978 & 0,978 & 0,977 & 0,977 \\
\hline
\end{tabular}

$\mathrm{a}=$ Um animal não atingiu convergência, neste caso.

A comparação dos modelos quanto ao ajustamento aos dados é apresentada na Tab. 5.

O modelo Gompertz foi o único no qual um animal não atingiu o critério de convergência. Quanto ao número de iterações, da menor para o maior, respectivamente, tem-se Brody, logístico, Von Bertalanffy e Gompertz (Tab. 5). Os valores obtidos neste estudo foram maiores do que os mencionados por Oliveira et al. (2000), porém o número de informações do presente estudo foi consistentemente maior. 
O quadrado médio do erro foi semelhante em todos os modelos, sendo o menor para o de Brody, seguido, em ordem crescente, por Von Bertalannfy, Gompertz e logístico. Em relação ao coeficiente de determinação, praticamente não houve diferença entre os modelos.

Nos componentes de variância estimados para os parâmetros da curva com maior significado biológico, houve ampla variação, o que confirma as estatísticas básicas apresentadas na Tab. 3 .
Os coeficientes de herdabilidade foram de elevada magnitude para os parâmetros estudados em todos os modelos (Tab. 6), indicando que poderiam ser usados como instrumento para alterar a forma da curva de crescimento desses animais, por seleção. A seleção ou o descarte com base no peso depois de o animal atingir a maturidade, poderá resultar em aumento do tamanho do animal adulto.

Tabela 6. Variâncias genética $\left(\sigma_{a}^{2}\right)$ e de ambiente $\left(\sigma_{e}^{2}\right)$ e herdabilidade $\left(h^{2}\right)$ dos pesos assintóticos (A) e taxa de maturação $(K)$ para cada modelo, obtidas a partir da análise unicaráter em fêmeas da raça Nelore

\begin{tabular}{lcccc}
\hline \multirow{2}{*}{ Modelo } & Parâmetro & $\sigma_{a}{ }^{2}$ & $\sigma_{e}$ & $h^{2}$ \\
\hline \multirow{2}{*}{ Von Bertalanffy } & $\mathrm{A}$ & 1225,04 & 1943,19 & 0,39 \\
\multirow{2}{*}{ Brody } & $\mathrm{K}^{1}$ & 91,52 & 128,45 & 0,42 \\
& $\mathrm{~A}$ & 2710,11 & 3670,55 & 0,42 \\
\multirow{2}{*}{ Logístico } & $\mathrm{K}^{1}$ & 55,17 & 70,20 & 0,44 \\
& $\mathrm{~A}$ & 1044,91 & 1577,35 & 0,40 \\
\multirow{2}{*}{ Gompertz } & $\mathrm{K}^{1}$ & 138,65 & 197,35 & 0,41 \\
& $\mathrm{~A}$ & 1103,51 & 1711,87 & 0,39 \\
\hline
\end{tabular}

${ }^{1} \times 10^{-3}$.

Os valores de herdabilidade estimados neste trabalho são menores do que os publicados por Duarte (1975), Ludwig (1977) e Oliveira (1995) para os modelos Brody e Von Bertalanffy, e superiores aos estimados por Ludwig (1977) e Duarte (1975) para os modelos logístico e Gompertz. Também foram superiores aos coeficientes estimados por Carrijo (1988), que utilizou o modelo Von Bertalanffy.

Os coeficientes de herdabilidade estimados para $\mathrm{K}$ foram superiores aos citados para a raça Nelore e zebuínos em geral (Duarte, 1975; Ludwig, 1977; Oliveira, 1995), sendo mais próximos aos encontrados para o gado taurino (Brown et al., 1972; DeNise e Brinks, 1985; Jenkins et al., 1991).

A partir da análise bicaráter, foram obtidos os valores de covariância e correlação genética apresentados na Tab. 7. Os coeficientes de herdabilidade e variância mantiveram os mesmos valores estimados na análise unicaráter.
Tabela 7. Covariâncias ( $\sigma)$ e correlações genéticas $\left(\mathrm{r}_{\mathrm{g}}\right)$ entre os pesos assintóticos e taxa de maturação para cada modelo a partir da análise bicaráter em fêmeas da raça Nelore

\begin{tabular}{lcc}
\hline Modelo & $\sigma$ & $\mathrm{r}_{\mathrm{g}}$ \\
\hline Von Bertalanffy & $-265,94$ & $-0,69$ \\
Brody & $-179,28$ & $-0,52$ \\
Logístico & $-184,80$ & $-0,56$ \\
Gompertz & $-183,85$ & $-0,49$ \\
\hline
\end{tabular}

A correlação genética entre os dois parâmetros é alta e negativa, semelhante às encontradas na literatura para zebuínos (Duarte, 1975; Carrijo, 1988; Oliveira, 1995), indicando que variações genéticas que resultam em aumento no peso assintótico também provocam redução na taxa de maturação dos animais.

A possibilidade de se alterar a forma da curva de crescimento em uma raça pela seleção dos parâmetros de algum modelo, segundo Fitzhugh (1976), depende da variância genética aditiva de cada parâmetro do modelo e da independência entre eles. Ambos apresentam alta variabilidade genética, mas forte ligação (Tab. 6 e 7). Mesmo 
assim, seria possível levar em consideração o peso à maturidade, juntamente com outras características, no descarte dos animais adultos do rebanho. Assim, o peso à maturidade seria controlado e, conseqüentemente, a taxa de maturação seria aumentada.

Os resultados, em conjunto, indicam que qualquer um dos modelos estudados pode ser utilizado para descrever a curva de crescimento de fêmeas da raça Nelore. Contudo, o modelo Brody foi o que melhor se ajustou aos dados observados, descreveu o peso assintótico das fêmeas e apresentou os menores números de iterações e quadrado do erro, com elevado coeficiente de determinação.

\section{CONCLUSÕES}

Os quatro modelos estudados (Von Bertalanffy, Brody, Gompertz e logístico) foram apropriados para estabelecer padrões médios de crescimento de fêmeas da raça Nelore. Levando-se em consideração os aspectos de facilidade computacional e precisão de ajuste aos dados observados, os melhores resultados foram proporcionados pelo modelo Brody. Em função dos parâmetros genéticos estimados para o peso assintótico e para a taxa de maturação, essas características são passíveis de ser incluídas em um índice de seleção.

\section{AGRADECIMENTOS}

Os autores agradecem aos criadores do PMGRN, às entidades de fomento à pesquisa: FINEP/BID, CNPq/RHAE, FAPESP e CAPES.

\section{REFERÊNCIAS BIBLIOGRÁFICAS}

ARANGO, J.A.; VAN VLECK, L.D. Size of beef cows: early ideas, new developments. Gen. Mol. Res., v.1, p.51-63, 2002.

BELTRAN, J.; BUTTS, W.; OLSON, T. et al. Growth patterns of two lines of Angus cattle selected using predicted growth parameters. $J$. Anim. Sci., v.70, p.734-741, 1992.

BOLDMAN, K.G.; KRIESE, L.A.;VAN VLECK, L.D. et al. A manual for use of MTDFREML: a set of programs to obtain estimates of variance and covariance [DRAFT]. Lincoln: Agricultural Research Service, 1995. 120 p.

BROWN, J.E.; FITHUGH JUNIOR, H.A.; CARTWRIGHT, T.C. A comparison of nonlinear models for describing weight-age relationships in cattle. J. Anim. Sci., v.42, p.810818, 1976.

CARRIJO, S.M. Descrição e comparação de parâmetros de crescimento de animais das raças Chianina e Nelore. 1988. Dissertação (Mestrado) - Universidade de São Paulo, Ribeirão Preto, SP.

CARTWRIGHT, T.C. Selection criteria for beef cattle for the future. J. Anim. Sci., v.30, p.706711, 1970.

CORTARELli, A.; DUARTE, F.A.M.; LÔBO, R.B. Ajuste do modelo Gompertz a dados de crescimento de bezerros da Raça Nelore. Cientifica, v.11, p.1-8, 1983.

DENISE, K.R.S.; BRINKS, J.S. Genetic and environmental aspects of the growth curve parameters in beef cows. J. Anim. Sci., v.61, p.1431-1440, 1985.

DUARTE, F.A.M. Estudo da curva de crescimento de animais da raça Nelore, através de cinco modelos estocásticos. 1975. 284f. Tese (Livre Docência) - Universidade de São Paulo, Ribeirão Preto, SP.

ELER, J.P.; SILVA, J.A.II V.; FERRAZ, J.B.S. et al. Genetic evaluation of the probability of pregnancy at 14 months for Nellore heifers. $J$. Anim. Sci., v.80, p.951-954, 2002.

FITZHUGH Jr., H.A. Analysis of growth curves and strategies for altering their shape. J. Anim. Sci., v.42, p.1036-151, 1976.

JENKINS, T.G.; KAPS, M.; CUNDIFF, L.V. et al. Evaluation of between -and within- breed variation in measures of weight-age relationships. J. Anim. Sci., v.69, p.3118-3128, 1991.

KAPS, M.; HERRING, W.O.; LAMBERSON, W.R. Genetic and environmental parameters for traits derived from the Brody growth curve and their relationships with weaning weight in Angus cattle. J. Anim. Sci., v.78, p.1436-1442, 2000.

LANNA, D.P.; PACKER, I.U. A produtividade da vaca Nelore. In: SIMPÓSIO: O NELORE DO 
SÉCULO XXI, 4., 1997, Uberaba. Anais... Uberaba, 1997. p.73-86.

LUDWIG, A. Ajustamentos de curvas exponenciais ao crescimento de gado Nelore e análise de seus parâmetros. 1977. 84f. Dissertação (Mestrado) - Universidade Federal de Viçosa, Viçosa, MG.

NADARAJAH, K.; MARLOWE, T.J.; NOTTER, D.R. Growth patterns of Angus Charolais, Charolais $\times$ Angus and Holstein $\times$ Angus cows from birth to maturity. J. Anim. Sci., v.59, p.957-966, 1984.

OLIVEIRA, H.N. Análise genético-quantitativa da curva de crescimento de fêmeas da raça Guzerá. 1995. 73f. 1995. Tese (Doutorado) Universidade de São Paulo, Ribeirão Preto, SP.

OLIVEIRA, H.N.; LÔBO, R.B.; PEREIRA, C.S. Comparação de modelos não lineares para descrever o crescimento de fêmeas da raça Guzerá. Pesq. Agropec. Bras., v.35, p.18431851, 2000.
PEROTTO, D.; CUE, R.I.; LEE, A.J. Comparison of nonlinear functions for describing the growth curve of three genotypes of dairy cattle. Can. J. Anim. Sci., v.72, p.773-782, 1992.

ROSA, A.N.; LÔBO, R.B.; OLIVEIRA, H.N. et al. Peso adulto de matrizes em rebanhos de seleção da raça Nelore no Brasil. Rev. Bras. Zootec., v.30, supl.1, p.1027-1036, 2001.

SAMPAIO, I.B.M. Estatística aplicada à experimentação animal. Belo Horizonte: FEPMVZ, 1998. 221p.

SANTORO, K.R.; BARBOSA, S.B.P. Parâmetros de curvas de crescimento de bovinos Nelore no Estado de Pernambuco. In: REUNIÃO ANUAL DA SOCIEDADE BRASILEIRA DE ZOOTECNIA, 38., 2001, Piracicaba, Anais... Piracicaba: FEALQ, 2001. p.530-531.

STATISTICAL Analysis System. 6.12 versão para Windows ${ }^{\circledR}$. Cary: SAS Institute, 1996.

STEWART, T.S.; MARTIN, T.G. Optimal mature size of Angus cow productivity. Anim. Prod., v.37, p.179-182, 1983. 